\title{
Resolvent Energy and Estrada Index of Benzenoid Hydrocarbons
}

\author{
Izudin Redžepović ${ }^{1}$, Boris Furtula ${ }^{1^{*}}$ \\ ${ }^{1}$ Faculty of Science, University of Kragujevac, P. O. Box 60, 12 Radoja Domanovica Street, \\ 34000 Kragujevac, Serbia \\ e-mail: izudin.redzepovic@pmf.kg.ac.rs, boris.furtula@pmf.kg.ac.rs \\ *corresponding author
}

\begin{abstract}
The relationship between the resolvent and Estrada indices for the alkanes has been recently demonstrated. That relationship involved the first Zagreb index besides these two eigenvaluebased molecular descriptors. In this paper, the quality of the correlation is tested in the case of isomeric benzenoid hydrocarbons, where the first Zagreb index is constant. Extraordinary linear correlations are identified for all studied groups of isomeric benzenoid hydrocarbons. Additionally, the relationship of these indices with the boiling points of a set of benzenoid hydrocarbons is presented.
\end{abstract}

Keywords: molecular descriptors, eigenvalues, adjacency matrix, computational modeling, molecular structure

\section{Introduction}

Information, stored within the molecular structure, is usually accounted for determining physico-chemical features of the corresponding compound. Extracting this information represents an important task when it comes to the modeling of molecular physico-chemical properties. This is ordinarily done by using molecular descriptors (Todeschini and Consonni 2009). Nowadays, there are a lot of such descriptors, and their number is still increasing. The most prominent group of molecular descriptors are those derived from 2D molecular structure, namely, topological descriptors. Transforming structural information into a topological index causes the loss of pieces of information carried by the molecular structure. Nevertheless, topological descriptors found remarkable applications in diverse fields of chemistry (Dudek et al. 2006, Mauri et al. 2017). One of the most common utilization of the topological indices is in QSPR/QSAR modeling (Devillers and Balaban 1999, Talevi et al. 2012, Roy et al. 2015, Zanni et al. 2015, Dearden 2017).

The graph theory found a significant application in chemistry through the use of topological descriptors. Namely, in chemical graph theory, a molecule is represented by the corresponding graph $G$ in which vertices stand for non-hydrogen atoms and edges for bonds. Topological indices are also known as graph invariants. They can be computed from the molecular graph by applying different algorithms. Thus, depending on what parameters are used 
in their definitions one can distinguish degree-, distance-, and eigenvalue-based topological molecular descriptors.

The eigenvalue-based descriptors became popular mathematical quantities since the explanation of the physical meaning of the eigenvalues that occurs in the HMO theory (Cvetković et al. 1972). These invariants are obtained using the eigenvalues from one of the graph matrices (usually from adjacency matrix). The very first such an index is the graph energy. It is defined in the following way:

$$
E(G)=\sum_{i=1}^{n}\left|\lambda_{i}\right|
$$

where $n$ stands for the number of vertices and $\lambda_{i}$ for the $i$-th eigenvalue of the graph $G$.

This quantity is attracting a lot of attention from both, theoretical chemists and mathematicians. Many papers and books are dealing with this index, see for example (Li et al. 2012) and references cited therein.

Another popular topological eigenvalue-based molecular descriptor is the Estrada index (Estrada 2000). It was introduced as a tool for capturing information on folding in biomolecules. It is defined as:

$$
E E(G)=\sum_{i=1}^{n} e^{\lambda_{i}}
$$

Recently, a novel index based on the "ordinary" eigenvalues was established. In the paper Gutman et al. (2016), eigenvalue-based graph invariant based on the eigenvalues of the resolvent matrix was proposed. It was named resolvent energy, and it is defined in the following way:

$$
E R(G)=\sum_{i=1}^{n} \frac{1}{n-\lambda_{i}}
$$

where $\lambda_{i}$ for the $i$-th eigenvalue of an adjacency matrix of the graph $G$.

In our recent paper (Redžepović and Furtula 2020) we have established an approximate relation connecting resolvent energy and Estrada index in the case of alkanes (i.e. chemical trees $T)$, Eq. 4 .

$$
E R(T) \approx \frac{\alpha}{n} E E(T)-\frac{\alpha n^{4}-24}{12 n^{5}} Z g_{1}(T)-\frac{11 \alpha n^{4}-24 n^{2}+24}{12 n^{5}}(n-1)-\alpha-1
$$

where $\alpha, n$, and $Z g_{1}$ stand for the fitting parameter, the number of vertices, and for the first Zagreb index, respectively.

The first Zagreb index $\left(Z g_{1}\right)$ is the degree-based molecular descriptor that was introduced years ago (Gutman and Trinajstić 1972) and is defined as:

$$
Z g_{1}(G)=\sum_{i=1}^{n} d_{i}^{2}
$$

where $d_{i}$ denotes the degree (i.e. the number of first neighbors) of the $i$-th vertex of the graph $G$.

It was shown that in the case of alkanes resolvent energy can be successfully modeled by Eq. 4, i.e. by Estrada index and first Zagreb index. The aim of this study is twofold. Firstly, it 
will be demonstrated a reasonable correlation between these indices with the boiling points of a set of benzenoid hydrocarbons. Then, a possible correlation between resolvent energy and the Estrada index in the case of isomeric benzenoid hydrocarbons. The benzenoid hydrocarbons are a large group of compounds that are subject to numerous investigations. More on mathematical and chemical treatment of benzenoid hydrocarbons can be found in (Gutman 1974, Gutman and Cyvin 1989, Gutman 2003, Allison and Burgess 2015, Redžepović et al. 2019), and references cited therein.

\section{Results and discussion}

\subsection{Estrada index and resolvent energy vs. boiling points of benzenoid hydrocarbons}

Experimental assessing of the physico-chemical properties of benzenoid hydrocarbons is cumbersome because of their unusual thermodynamic stability. Thence, there are no much data on physico-chemical properties of benzenoid hydrocarbons. Nevertheless, we implied here dataset consisting of 21 boiling points of benzenoid hydrocarbons to picture a vague idea on the predictive potential of the Estrada index and the resolvent energy.

The predictive power of these two molecular descriptors was tested on modeling the boling points of benzenoid hydrocarbons, both cata- and pericondensed, listed in Table 1. Boiling points were drawn from the paper Nikolić et al. (2004). The calculated boiling points were obtained by simple linear model:

$$
\text { boiling point } \approx a \times T I+b
$$

where TI is Estrada index or resolvent energy, and $a$ and $b$ are the coefficients obtained by least square fitting. To calculate $a$ and $b$ simple Python script was written using scikit-learn module (Pedregosa et al. 2011). For model based on ER $a=-21556.52$ and $b=22195.21$, and for model based on $E E$ a $=9.01$ and $b=8.63$.

\begin{tabular}{|l|c|}
\hline molecule & BP $\left({ }^{\circ} \mathbf{C}\right)$ \\
\hline Naphthalene & 218 \\
\hline Phenanthrene & 338 \\
\hline Anthracene & 340 \\
\hline Tetraphene & 425 \\
\hline Triphenylene & 429 \\
\hline Chrysene & 431 \\
\hline Tetracene & 440 \\
\hline Benzo[e]pyrene & 493 \\
\hline Benzo[a]pyrene & 496 \\
\hline Perylene & 497 \\
\hline Picene & 519 \\
\hline Benzo[m]tetraphene & 531 \\
\hline Benzo[f]tetraphene & 535 \\
\hline Benzo[k]tetraphene & 536 \\
\hline Benzo[g,h,i]perylene & 542 \\
\hline Anthanthrene & 547 \\
\hline
\end{tabular}




\begin{tabular}{|l|l|}
\hline Coronene & 590 \\
\hline Dibenzo[a,e]pyrene & 592 \\
\hline Dibenzo[a,i]pyrene & 594 \\
\hline Dibenzo[a,l]pyrene & 595 \\
\hline Dibenzo[a,h]pyrene & 596 \\
\hline
\end{tabular}

Table 1. Experimental boiling points of bezenoid hydrocarbons applied for constructing the linear models.

In Fig. 1 is depicted corellation between experimental vaules from Table 1 and estimated boiling points by model based on Estrada index. With $82 \%$ of explained variance, this simple linear model shows satisfactory predictive potential. Model based on resolvent energy explains $84 \%$ od data variance. These findings indicate that these two eigenvalue-based descriptors have similar predictive power.

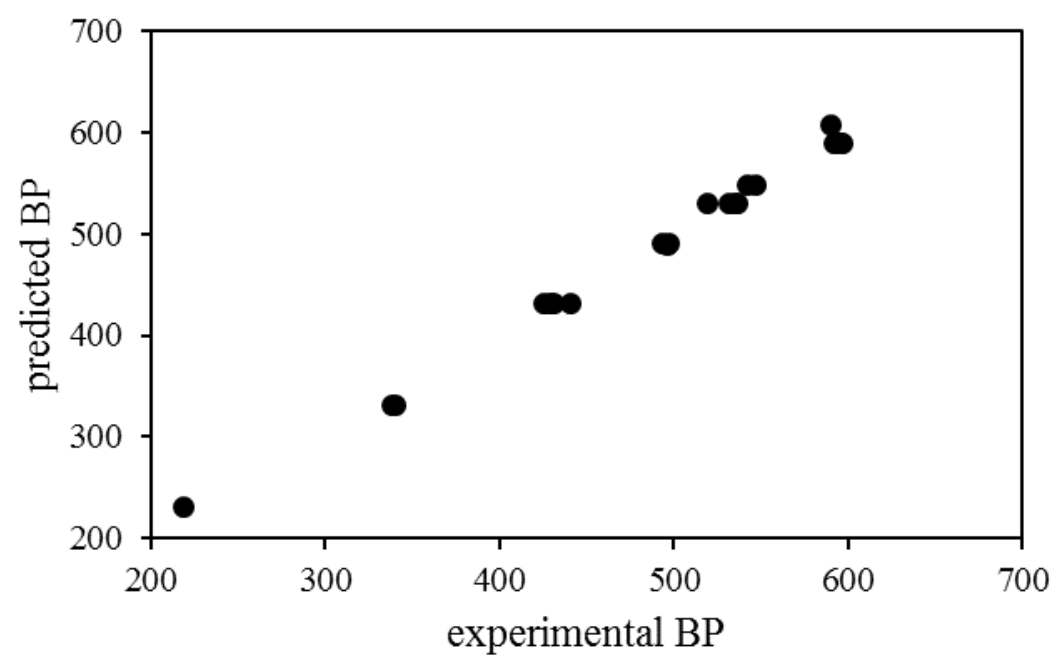

Fig 1. Scatter plot of experimental versus predicted boiling points of benzenoid hydrocarbons.

\subsection{Estrada index vs. resolvent energy of benzenoid hydrocarbons}

Benzenoid hydrocarbons can be divided into two groups: catacondensed and pericondensed molecules. The difference between them is in the fact that compounds in the latter group contain internal carbon atoms. Graphs obtained from benzenoid hydrocarbons contain only vertices with the degree 2 or 3 . The number of vertices of degree $2\left(n_{2}\right)$ and degree $3\left(n_{3}\right)$ can be obtained from the following expressions (Gutman 2003):

$$
\begin{gathered}
n_{2}=2 h+4-n_{1} \\
n_{3}=2 h-2
\end{gathered}
$$

where $h$ denotes the number of hexagons, and $n_{i}$ the number of internal vertices. In the group of isomeric benzenoid hydrocarbons, $h$ and $n_{i}$ are constant, implying that $Z g_{l}=$ const .

Based on Eq. 4, it is expected that among isomeric benzenoid hydrocarbons there is a linear correlation between the resolvent energy $(E R(B))$ and the Estrada index $(E E(B))$. Moreover, it 
suggests that in the case of isomers of benzenoid hydrocarbons, the $E R(B)$ solely depends on $E E(B)$. Since the range of values of these two indices differs significantly, and in order to eliminate the intercept of this linear correlation, a slightly modified normalization of data is done using the following formula:

$$
X_{i}^{\text {new }}=\frac{X_{i}-X_{\max }}{s(x)}
$$

After this rearrangement of data, the following relation between $E R(B)$ and $E E(B)$ is expected:

$$
E R(B) \approx E E(B)
$$

To test our assumptions, we have employed groups of the catacondensed molecules with the $h$ ranging from 5 up to 10 . As for the pericondensed molecules, $h$ ranged from 8 up to 10. It should be emphasized that within the group of pericondensed molecules with the same number of hexagons number of internal vertices is constant. A reason for performing tests on pericondensed benzenoid hydrocarbons with no less than 8 hexagons lies in the fact that there are just a few such compounds with the smaller $h$. Additionally, the pericondensed benzenoids, having 8-10 hexagons, with a smaller number of internal carbon atoms were omitted, because their number is one or two per $n_{i}$. All molecules in this paper are generated using the $\mathrm{CaGe}$ computer program (Brinkmann et al. 2010). The results are presented in Table 2.

\begin{tabular}{|l|l|c|c|c|}
\hline & $\mathbf{h}$ & \multicolumn{2}{|c|}{ \#isomers } & $\mathbf{R}$ \\
\hline \multirow{5}{*}{ acondensed } & 5 & \multicolumn{2}{|c|}{12} & 0.99990 \\
\cline { 2 - 5 } & 6 & \multicolumn{2}{|c|}{36} & 0.99978 \\
\cline { 2 - 5 } & 7 & \multicolumn{2}{|c|}{118} & 0.99981 \\
\cline { 2 - 5 } & 8 & \multicolumn{2}{|c|}{411} & 0.99986 \\
\cline { 2 - 5 } & 9 & \multicolumn{2}{|c|}{1489} & 0.99987 \\
\hline & 10 & \multicolumn{2}{|c|}{5572} & $\mathbf{R}$ \\
\hline \multirow{3}{*}{ icondensed } & $\mathbf{h}$ & \#isomers & 6 & 0.99994 \\
& 8 & 8 & 7 & 0.99997 \\
\cline { 2 - 5 } & 9 & 12 & 8 & 0.99992 \\
\cline { 2 - 5 } & 10 & 25 & & \\
\hline
\end{tabular}

Table 2. Correlation coefficients (R) between resolvent energy and Estrada index of benzenoid hydrocarbons

In Fig. 2 resolvent energy is plotted against the Estrada index in the case of catacondensed benzenoid hydrocarbons, and in Fig. 3 this correlation is depicted in the case of pericondensed benzenoid hydrocarbons. 


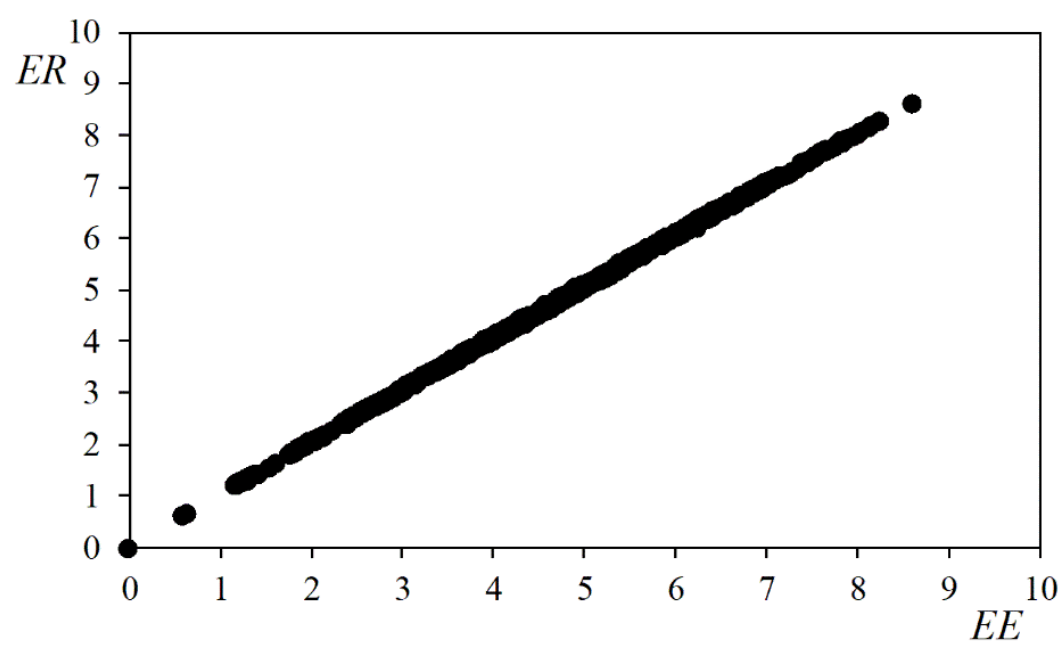

Fig 2. Normalized resolvent energy values plotted against normalized Estrada index for catacondensed benzenoid hydrocarbons with 10 hexagons

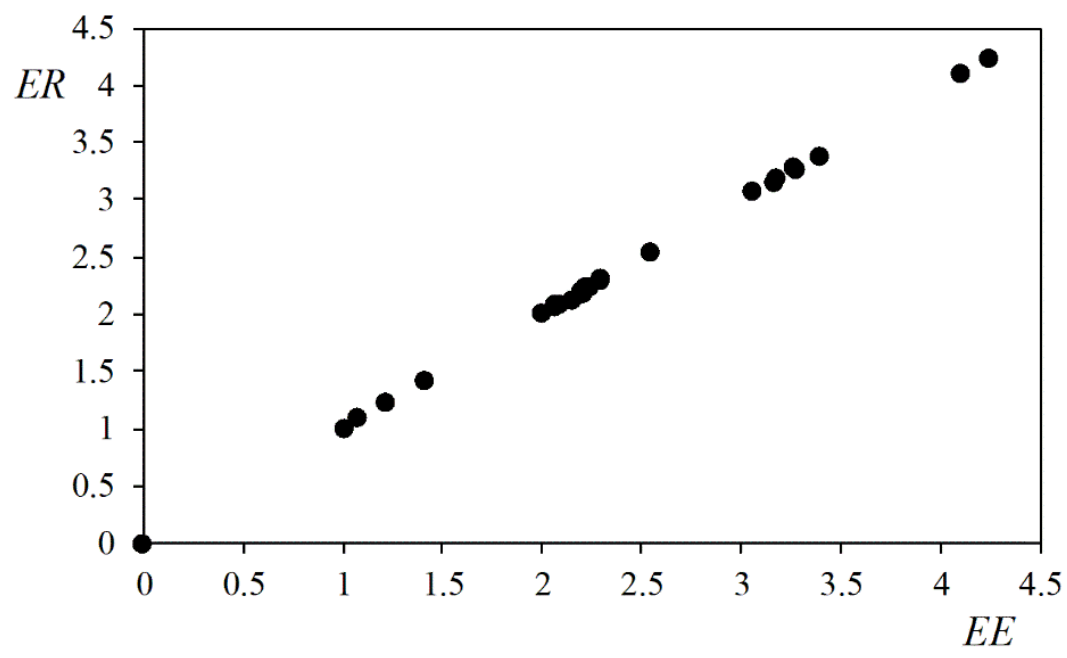

Fig 3. Normalized resolvent energy values plotted against normalized Estrada index for pericondensed benzenoid hydrocarbons with 10 hexagons

Figs. 2 and 3 show that there is a very good linear correlation between resolvent energy and Estrada index in isomeric benzenoid hydrocarbons. This finding indicates that mathematical approximative formula that relates resolvent energy and Estrada index (Eq. 4) is valid for isomeric benzenoid hydrocarbons as well. 


\section{Conclusions}

The resolvent energy and Estrada index are eigenvalue-based topological molecular descriptors. A natural way of thinking is that they may be correlated since the same parameters are used in their definitions. This assumption is proved and mathematically elaborated in the case of alkanes. The results of this paper further corroborate this correlation. Extraordinary linear relationships between resolvent energy and Estrada index are identified in benzenoid hydrocarbons, both in catacondensed and pericondensed molecules. This finding validates the approximative formula connecting the resolvent energy and the Estrada index, established in the paper (Redžepović and Furtula 2020). Also, these two descriptors show similar predictive potential in the case of benzenoid hydrocarbons. This implies that only one of these indices should be used in QSPR studies because both of them carry the very same information on the structure of a benzenoid hydrocarbon.

Acknowledgments This work was supported by the Ministry of Education, Science and Technological Development of the Republic of Serbia through the grant no. 174033.

\section{References}

Allison TC, Burgess DR (2015). First-principles prediction of enthalpies of formation for polycyclic aromatic hydrocarbons and derivatives. Journal of Physical Chemistry A. 119: 11329-11365.

Brinkmann G, Delgado Friedrichs O, Lisken S, Peeters A, Van Cleemput N (2010). CaGe-a virtual environment for studying some special classes of plane graphs-an update. MATCH Communications in Mathematical and in Computer Chemistry. 63: 533-552.

Cvetković D, Gutman I, Trinajstić N (1972). Graph Theory and Molecular Orbitals. II. Croatica Chemica Acta. 44: 365-374.

Dearden CJ (2017). Advances in QSAR modeling-applications in pharmaceutical, chemical, food, agricultural and environmental sciences (K Roy). Springer, Cham, Switzerland.

Devillers J, Balaban AT (1999). Topological indices and related descriptors in QSAR and QSPR. Gordon \& Breach, Amsterdam, The Netherlands.

Dudek A, Arodz T, Galvez J (2006). Computational methods in developing quantitative structure-activity relationships (QSAR): A review. Combinatorial Chemistry \& High Throughput Screening. 9: 213-228.

Estrada E (2000). Characterization of 3D molecular structure. Chemical Physics Letters. 319: $713-718$.

Gutman I (1974). Some topological properties of benzenoid systems, Croatica Chemica Acta. 46: 209-215.

Gutman I, Cyvin CJ (1989). Introduction to the theory of benzenoid hydrocarbons. Springer, Berlin, Germany.

Gutman I (2003). Uvod u hemijsku teoriju grafova. Prirodno-matematički fakultet, Kragujevac, Srbija.

Gutman I, Furtula B, Zogić E, Glogić E (2016). Resolvent energy of graphs. MATCH Communications in Mathematical and in Computer Chemistry. 75: 279-290.

Gutman I, Trinajstić N (1972). Graph theory and molecular orbitals. Total $\pi$-electron energy of alternant hydrocarbons. Chemical Physics Letters. 17: 535-538.

Li X, Shi Y, Gutman I (2012). Graph energy. Springer, New York, USA.

Mauri A, Consonni V, Todeschini R (2017). Handbook of Computational Chemistry (Eds. J. Leszczynski, A Kaczmarek-Kedziera, T Puzyn, GM Papadopoulos, H Reis, KM Shukla). Springer, Cham, Switzerland. 
Nikolić S, Miličević A, Trinajstić N, Jurić A (2004). On use of the variable Zagreb ${ }^{\mathrm{v}} \mathrm{M}_{2}$ index in QSPR: boiling points of benzenoid hydrocarbons. Molecules. 9: 1208-1221.

Pedregosa F, Varoquaux G, Gramfort A, Michel V, Thirion B, Grisel O, Blondel M, Prettenhofer P, Weiss R, Dubourg V, Vanderplas J, Passos A, Cournapeau D, Brucher M, Perrot M, Duchesnay E (2011). Journal of Machine Learning Research. 12: 2825-2830.

Redžepović I, Furtula B (2020). On relationships of eigenvalue-based topological molecular descriptors. Acta Chimica Slovenica. 67: 312-318.

Redžepović I, Marković S, Furtula B (2019). On structural dependence of enthalpy of formation of catacondensed benzenoid hydrocarbons. MATCH Communications in Mathematical and in Computer Chemistry. 82: 663-678.

Roy K, Kar S, Das NR (2015). A primer on QSAR/QSPR modeling-fundamental concepts. Springer, Cham, Switzerland.

Talevi A, Bellera CL, Ianni DM, Duchowicz RP, Bruno-Blanch EL, Castro AE (2012). An integrated drug development approach applying topological descriptors. Current Computer-Aided Drug Design. 8: 172-181.

Todeschini R, Consonni V (2009). Molecular descriptors for chemoinformatics. Wiley-VCH, Weinheim, Germany.

Zanni R, Galvez-Llompart M, García-Domenech R, Galvez J (2015). Latest advances in molecular topology applications for drug discovery. Expert Opinion on Drug Discovery. 10: 945-957. 